\title{
Ischemic stroke after use of the synthetic marijuana "spice"
}

Melissa J. Freeman, MD

David Z. Rose, MD

Martin A. Myers, MD

Clifton L. Gooch, MD

Andrea C. Bozeman, MS, ARNP-C

W. Scott Burgin, MD

Correspondence to

Dr. Burgin:

wburgin@health.usf.edu

\section{ABSTRACT}

Objectives: To report and associate acute cerebral infarctions in 2 young, previously healthy siblings with use of the street drug known as "spice" (a synthetic marijuana product, also known as "K2"), which they independently smoked before experiencing acute embolic-appearing ischemic strokes.

Methods: We present history, physical examination, laboratory data, cerebrovascular imaging, echocardiogram, ECG, and hospital course of these patients.

Results: We found that in both siblings spice was obtained from the same source. The drug was found to contain the schedule I synthetic cannabinoid JWH-018. Full stroke workup was unrevealing of a stroke etiology; urine drug screen was positive for marijuana.

Conclusions: We found that our 2 patients who smoked the street drug spice had a temporal association with symptoms of acute cerebral infarction. This association may be confounded by contaminants in the product consumed (i.e., marijuana or an unidentified toxin) or by an unknown genetic mechanism. The imaging of both patients suggests an embolic etiology, which is consistent with reports of serious adverse cardiac events with spice use, including tachyarrhythmias and myocardial infarctions. Neurology ${ }^{\circledast 2}$ 2013;81:2090-2093

\section{GLOSSARY}

DEA = Drug Enforcement Agency; lg = immunoglobulin; INR = international normalized ratio; LDL = low-density lipoprotein; MCA = middle cerebral artery; $\mathbf{P T}=$ prothrombin time; $\mathbf{P T T}=$ partial thromboplastin time; $\mathbf{t}-\mathbf{P A}=$ tissue plasminogen activator; TSH $=$ thyroid stimulating hormone.

Spice, a schedule I synthetic marijuana (also known as "K2"), is a recreational street drug with many known adverse effects, including seizures and myocardial infarction. However, to our knowledge, cerebral infarction associated with synthetic marijuana use has yet to be reported in the medical literature. Spice is frequently abused by young adults with increasing popularity because of its euphoric effects and the ease with which it is obtained, which creates the perception of "a legal high.” The US Drug Enforcement Agency (DEA), law enforcement, hospitals, and poison control centers have noted gradually increasing use of spice in the United States since 2009. In 2011, 5 synthetic cannabinoids used in spice were categorized as schedule I substances under the Controlled Substances Act: JWH-018; JWH-073; JWH-200; CP-47,497; and cannabicyclohexanol. ${ }^{1}$ At the time of our patients' presentations, spice was available for sale over-the-counter at convenience stores, smoke shops, gas stations, and over the Internet, and labeled as "herbal blends," "air fresheners," or "incense," often with the warning "not for human consumption." Common side effects of spice include tachycardia, vomiting, agitation, confusion, and hallucinations; more serious adverse reactions (seizures, myocardial infarction) have recently appeared in the medical literature. ${ }^{2-4}$ Furthermore, a number of spice-associated deaths have been reported in national news media. To our knowledge, the medical literature has not yet reported ischemic stroke associated with synthetic marijuana. We present 2 young siblings who experienced acute ischemic strokes soon after smoking synthetic marijuana.

CASE REPORTS Patient A. A 26-year-old man was brought to the emergency department by emergency medical services approximately 50 minutes after he had the sudden onset of dysarthria, expressive aphasia, and right face

From the Department of Neurology, University of South Florida, Tampa.

Go to Neurology.org for full disclosures. Funding information and disclosures deemed relevant by the authors, if any, are provided at the end of the article. 
and arm weakness. At the time of arrival, he was anarthric. He had asthma, but no other significant medical history per family, and used no daily medications. $\mathrm{He}$ did not smoke tobacco or drink alcohol, and there was no family history of any blood clots, hypercoagulability, congenital heart disease, arrhythmias, stroke, or stroke risk factors. His blood pressure was 158/ $88 \mathrm{~mm} \mathrm{Hg}$ and ECG revealed sinus tachycardia at $110 \mathrm{bpm}$, which later resolved to a sinus bradycardia. Noncontrast head CT showed a hyperdense left middle cerebral artery (MCA). CT perfusion revealed a large area of penumbra without core infarction (figure $1 \mathrm{~A}$ ), and left MCA clot was confirmed by CT angiography (figure 1B), so he received IV tissue plasminogen activator ( $\mathrm{t}-\mathrm{PA}$ ). Urine drug screen was positive only for cannabinoids; he did endorse smoking marijuana, but not in the days leading up to his stroke. Glucose, hemoglobin A1c, partial thromboplastin time (PTT), prothrombin time (PT)/international normalized ratio (INR), thyroid stimulating hormone (TSH), and complete blood count with platelet count were all within normal limits. Low-density lipoprotein (LDL) was 121. His troponin level was 0.00 , creatinine phosphokinase was 101 , creatine kinase $\mathrm{MB}$ was 0.7 , myoglobin was 38.3, and B-natriuretic peptide was 28. After receiving $\mathrm{t}-\mathrm{PA}$, his symptoms resolved and he stated he smoked spice "a few hours prior" to his symptom onset and that he had smoked marijuana in the past, but denied recent use. He also denied the use of any other street drugs. A synthetic marijuana lab panel was not sent because he personally revealed that he smoked spice, and at the time of his hospitalization we were not aware such a panel existed. Brain MRI performed after t-PA confirmed distal left MCA distribution infarction (figure 1C). Conventional cerebral angiogram showed a complete occlusion of the proximal M1 segment with adequate leptomeningeal collaterals; flow from the anterior cerebral artery filled the entire MCA back to the distal M1 segment. There were no radiographic signs of vasculitis or other arterial disease. Transthoracic and transesophageal echocardiography, and full hypercoagulable panel (cardiolipin antibodies immunoglobulin [Ig] G, IgM, and IgA; factor V Leiden, antithrombin 3, lupus anticoagulant, protein $\mathrm{C}$ antigen and activity, and protein $S$ antigen and activity) were unremarkable. $\mathrm{He}$ improved clinically and did not return for his followup visit.

Patient B. A 19-year-old woman with a history of tobacco use, anxiety disorder, and panic attacks presented with loss of consciousness and vomiting "a few minutes" after smoking spice, followed by several hours of persistent altered mental status and "shaking movements" of the arms and legs, according to witnesses. Upon hospital arrival, she had global aphasia, right hemiplegia, and sensory loss. Blood pressure was $143 / 73 \mathrm{~mm} \mathrm{Hg}$ with sinus tachycardia at 120 bpm. Urine drug screen was positive only for cannabinoids; it is unknown when she had smoked marijuana last because of her aphasia. CT angiogram showed an irregular contour of the proximal M1 segment of the left MCA without evidence of dissection. Thrombus was noted in the insular branches, suggestive of an embolic phenomenon (figure 2A). MRI revealed a large left MCA distribution infarction with punctate infarcts in the right cerebral hemisphere suggestive of a proximal embolic event (figure 2B). She did not arrive within the window for t-PA. TSH, Alc, complete blood count, and PT/PTT/INR were normal; LDL was 117. She had mild troponinemia without ECG changes. Bilateral leg ultrasound and pelvic MRI showed no deep venous thrombosis; transthoracic and transesophageal echocardiograms and hypercoagulable panel (factors 2, 5, 9; von Willebrand factor 8, antithrombin 3 , lupus anticoagulant, protein $\mathrm{C}$ antigen and activity, and protein $S$ antigen and activity) were unremarkable. Because we recognized this patient as the sibling who had visited her brother during his

Figure $1 \quad$ Patient A imaging

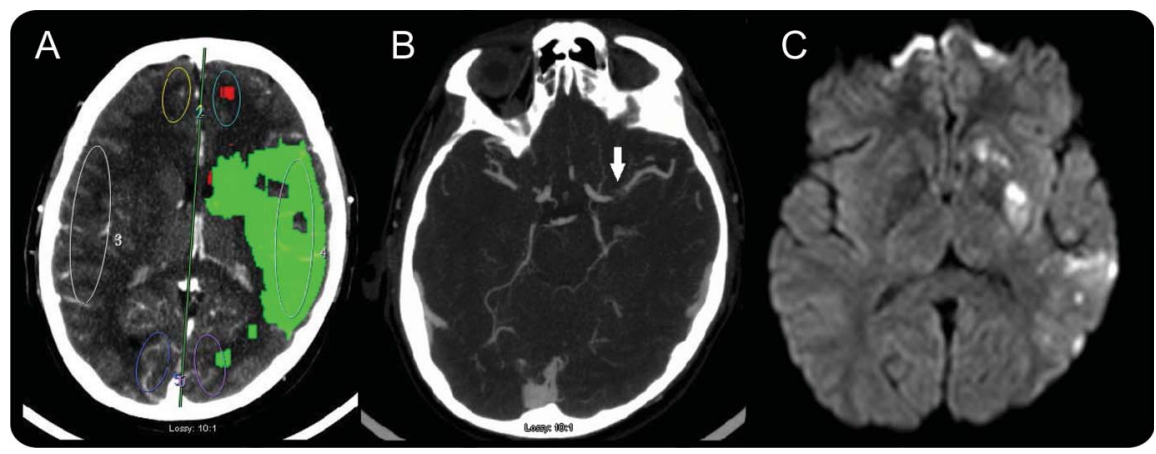

(A) Pre-t-PA CT perfusion of the brain with large area of penumbra in the left MCA territory. (B) Pre-t-PA CT angiogram shows proximal left MCA clot (arrow). (C) Post-t-PA MRI diffusion-weighted imaging sequence showing small areas of infarct in the left MCA distribution. MCA = middle cerebral artery; t-PA = tissue plasminogen activator. 
Figure 2 Patient B imaging

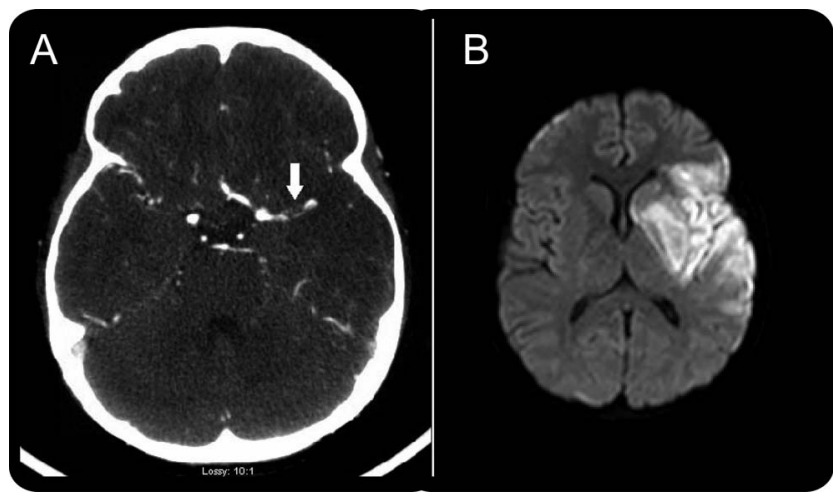

(A) CT angiogram showing a proximal left MCA clot (arrow). (B) MRI diffusion-weighted imaging sequence showing large area of infarct in the left MCA distribution. MCA = middle cerebral artery.

admission previously, and because it was noted that the spice they both smoked was from the same batch, we consulted toxicology staff who sent for synthetic cannabinoid metabolite screening (liquid chromatography-tandem mass spectroscopy from a serum sample). This was positive for JWH-018 and negative for the other testable compounds (AM-2201, JWH-019, JWH-073, JWH-250). She stabilized neurologically, but right hemiparesis and expressive aphasia remained at follow-up office visit.

DISCUSSION Spice has been consumed worldwide since at least the early $2000 \mathrm{~s},{ }^{2}$ rising to the attention of law enforcement, poison controls, and the DEA in the United States by 2009. With purported euphoric effects and minimal legal restrictions or regulations, it has seen increased demand from current marijuana smokers, recreational drug users, and curious experimenters who are drug-naive. ${ }^{2}$ One study found that approximately $11 \%$ of high school seniors admitted to using spice in $2012 .^{5}$ To date, there have been scant case reports of seizures and myocardial infarction with use of spice. ${ }^{2-4}$ Other abreactions from spice include supraventricular tachycardia, suicide, diaphoresis, and psychosis. ${ }^{4,6}$ Specifically, the compound JWH-018 has been reported to cause hallucinations, vertigo, paresthesias, shivering, shaking, tachycardia, extrasystoles, hypertension, nausea, vomiting, dry mouth, mydriasis, conjunctival hyperemia, and hypokalemia. ${ }^{3} \mathrm{JWH}-018$ is a full agonist to the cannabinoid receptors $\mathrm{CB} 1$ and $\mathrm{CB} 2$ and its activity at synaptic endings is well understood; however, the broad neurobiological and neurodevelopmental roles of endocannabinoid systems are less elucidated. A typical urine drug screen, able to identify marijuana, will not detect synthetic cannabinoids. Thus, if spice use is suspected, liquid chromatography-tandem mass spectroscopy from a serum sample may help confirm JWH-018, AM-2201, JWH-019, JWH-073,
JWH-250, and others. ${ }^{7.8}$ Nevertheless, the formula of spice varies by supplier, and can include such toxic chemicals as linoleic acid, palmitic acid, oleamide, palmitoylethanolamide, eugenol, thymol, acetyl vanillin, benzyl benzoate, $\alpha$-tocopherol, vitamin $\mathrm{E}$, and $\beta_{2}$-adrenergic agonists. ${ }^{2}$ Although the 5 cannabinoids are schedule I drugs as of 2011, the manufacturing of the street drugs is not currently regulated; the manufacturers are often unknown because these products are purchased through internet vendors both wholesale and retail. ${ }^{9}$ Moreover, to evade legal restrictions, manufacturers continue to modify these chemicals and create new ones, providing an ongoing market for their sale as the DEA continues to monitor the need to update the list of banned cannabinoids. Because they are not regulated and several manufacturers reside in other countries, ${ }^{9}$ manufacturers are not required to list all compounds on the label, making the synthetic drug an endless, rotating mystery of ingredients. Since the time of our patients' use of spice, the Florida Attorney General has made it a third degree felony to sell, manufacture, deliver, or possess with intent to sell these drugs.

Although at this point the strokes in our patients do remain cryptogenic, the appearance of a large clot in the M1 segment of patient $A$ and thrombi in multiple insular branches found in patient $\mathrm{B}$ - as well as the large vessel territories restricting on diffusionweighted brain MRI-all suggest an embolic etiology. This, coupled with previous reports of serious adverse cardiac events such as tachyarrhythmias and myocardial infarctions with spice consumption, suggests the possibility of a cardioembolic etiology, especially because of the lack of any cervical or intracranial arterial pathology to explain alternate mechanisms.

While both patients tested positive for regular cannabis and had smoked conventional marijuana in the past, it was JWH-018-confirmed spice that was smoked shortly before the stroke onset; per patient report, each obtained spice from the same supplier. Limitations to our report include the possibility that the spice was not the etiology of these siblings' strokes; an unidentified genetic mechanism was responsible given their relation; that the timing, use, and identical supplier of spice to each patient was coincidental; that unidentified toxins in the spice provoked their strokes; that they experienced a synergistic toxic effect of natural marijuana with its synthetic counterpart JWH-018; or that the etiology was marijuana itself. Although cannabis use is not conventionally associated with stroke, emerging case reports describe cerebral infarction with significant marijuana intake, ${ }^{10}$ especially after myocardial infarction and typically soon after smoking the marijuana. Uncoincidentally, the increase in interest in marijuana-related stroke has occurred simultaneously 
with an increase in the use of spice, which is undetectable in conventional toxicologic studies.

Neurologists, internists, emergency department personnel, and first responders should know of spice's association with seizures, myocardial infarction, and now possibly ischemic stroke. Careful history of spice use should be explored with unexplained ischemic events or focal neurologic deficits. Urine drug screens are helpful, but may not always capture newer compounds, and specific synthetic marijuana testing is helpful. Government agencies and legislators should continue to develop strategies to properly identify and regulate these potentially hazardous compounds.

\section{AUTHOR CONTRIBUTIONS}

Melissa Freeman, MD, David Rose, MD, Martin Myers, MD, Clifton Gooch, MD, Andrea Bozeman, ARNP, and Scott Burgin, MD: drafting/revising the manuscript for content.

\section{STUDY FUNDING}

No targeted funding reported.

\section{DISCLOSURE}

M. Freeman reports no disclosures. D. Rose is on the speakers bureau for Boehringer Ingelheim. M. Myers, C. Gooch, A. Bozeman, and W. Burgin report no disclosures. Go to Neurology.org for full disclosures.

Received May 15, 2013. Accepted in final form August 14, 2013.

\section{REFERENCES}

1. Harris CR, Brown A. Synthetic cannabinoid intoxication: a case series and review. J Emerg Med 2013;44:360-366.

2. Seely KA, Lapoint J, Moran JH, Fattore L. Spice drugs are more than harmless herbal blends: a review of the pharmacology and toxicology of synthetic cannabinoids. Prog Neuropsychopharmacol Biol Psychiatry 2012;39:234-243.

3. Hermanns-Clausen M, Kneisel S, Szabo B, Auwärter V. Acute toxicity due to the confirmed consumption of synthetic cannabinoids. Addiction 2012;108:534-544.

4. Pant $S$, Deshmukh A, Dholaria B, et al. Spicy seizure. Am J Med Sci 2012;344:67-68.

5. Vandrey R, Dunn KE, Fry JA, Girling ER. A survey study to characterize use of spice products. Drug Alcohol Depend 2012;120:238-241.

6. Wells DL, Ott CA. The new marijuana. Ann Pharmacother 2011;45:414-417.

7. Hudson BD, Hébert TE, Kelly ME. Ligand- and heterodimer-directed signaling of the $\mathrm{CB}(1)$ cannabinoid receptor. Mol Pharmacol 2010;77:1-9.

8. Teske J, Weller JP, Fieguth A, et al. Sensitive and rapid quantification of the cannabinoid receptor agonist naphthalene-1-yl-(1-pentylindol-3-yl) methanone (JWH-018) in human serum by liquid chromatography-tandem mass spectroscopy. J Chromatogr B Analyt Technol Biomed Life Sci 2010;878:2659-2663.

9. DEA. K2 \& spice fact sheet. Available at: http://www. justice.gov/dea/druginfo/drug_data_sheets/K2_Spice.pdf. Accessed July 2, 2013.

10. Wolff V, Armspach JP, Lauer V, et al. Cannabis-related stroke: myth or reality? Stroke 2013;44:558-563.

\section{Neurology ${ }^{\circledR}$ Launches Subspecialty Alerts by E-mail!}

Customize your online journal experience by signing up for e-mail alerts related to your subspecialty or area of interest. Access this free service by visiting http://www.neurology.org/site/subscriptions/etoc.xhtml or click on the "E-mail Alerts" link on the home page. An extensive list of subspecialties, methods, and study design choices will be available for you to choose from-allowing you priority alerts to cutting-edge research in your field!

\section{Commenting Online is Easier Now with WriteClick ${ }^{\mathrm{TM}}$}

Have a comment on a recent $N e u r o l o g y{ }^{\circledR}$ article you would like to share? Now it is easier and more convenient. Neurology.org has launched WriteClick on the home page and sidebars of each article to encourage remarks and debate among users.

WriteClick is restricted to comments about studies published in Neurology within the last eight weeks.

Learn more at http://www.neurology.org/letters 